\title{
Effect of Twist and Raise Walking Technique on ICU-acquired Weakness in COVID-19 Patients: A Pre-post Experimental Study
}

\author{
Prasannajeet Pramod Nikam* and Govindhan Varadharajulu
}

Department of Sports Physiotherapy, Faculty of Physiotherapy, Krishna Institute of Medical Sciences Deemed to be University, Karad - 415539, Maharashtra, India; prasannastaarc@gmail.com

\begin{abstract}
Background: Recent studies presenting first recommendations on respiratory rehabilitation management have failed to advocate a specific protocol as there is still missing data about the current specific needs due to COVID-19. This necessitates the extensive research to be carried out for investigating the efficacy of various physiotherapeutic interventional methods on these associated symptoms which often go unnoticed. In the same interest, an attempt was made to address the complications of prolonged immobilization by facilitating early ambulation along with incorporation of upper limb, lower limb as well as trunk movements. The aim was to facilitate chest expansion along with initiation of early ambulation in order to reduce the possible complications of ICU acquired weakness. Materials and Methods: 32 COVID-19 positive subjects were given the twist and raise walking technique as a rehabilitative intervention for a period of 7 days. Post intervention data for various outcome measures like inspiratory hold capacity and forced expiration were recorded. Results: Clinically significant results were seen in post-test scores of all the subjects in terms of Inspiratory Hold Capacity $(\mathrm{p}<0.0001)$ as well as Forced Expiration Volume $(\mathrm{p}<0.0001)$. Conclusion: Twist and Raise Walking Technique was proven significant in reducing the intensity of ICU-acquired weakness by improving both the inspiratory hold capacity as well as the forced expiration. This technique can be advocated as a effective rehabilitation intervention especially in COVID-19 patients to minimize the possible complications of ICU-acquired weakness.
\end{abstract}

Keywords: COVID-19, ICU Acquired Weakness, Physiotherapy, Rehabilitation

\section{Introduction}

Severe acute respiratory syndrome coronavirus 2 (SARSCoV-2) is a new coronavirus that emerged in 2019 and causes coronavirus disease 2019 (COVID-19) ${ }^{1,2}$. The symptoms of the disease include fever, headache, bodyache and dry cough. It is the associated symptoms of this disease like dysphagia, muscle weakness, critical illness myopathy and neuropathy, reduced joint mobility, neck and shoulder pain (due to prolonged proning), difficulty in verticalization, impaired balance and gait, difficulties in Activities of Daily Living (ADL), difficulty awakening with long-lasting confusional state and psychological problems which contribute to the deterioration of the rehabilitative process ${ }^{3}$. The common causative factor which can be linked to almost each of the above mentioned associated complications is the prolonged immobilization secondary to bed-rest.

As a result of the extensive medical management given to a COVID-19 patient which includes prolonged protective lung ventilation, sedation and use of neuromuscular blockading agents, COVID-19 patient admitted in the ICU might be anticipated to be at high risk for developing ICU acquired weakness ${ }^{4}$. While early attempts

*Author for correspondence 
are expected to be made by the physiotherapist directed towards reducing the disability and improving the respiratory parameters, there is no sequence or a specific protocol or technique which would specifically fulfill all the rehabilitation needs essential for the respiratory as well as musculoskeletal components of breathing.

Recent studies presenting first recommendations on respiratory rehabilitation management $t^{5-7}$ have failed to advocate a specific protocol as there is still missing data about the current specific needs due to COVID-19. This necessitates the extensive research to be carried out for investigating the efficacy of various physiotherapeutic interventional methods on these associated symptoms which often go unnoticed. In the same interest, an attempt was made to address the complications of prolonged immobilization by facilitating early ambulation along with incorporation of upper limb, lower limb as well as trunk movements. The aim was to facilitate chest expansion along with initiation of early ambulation in order to reduce the possible complications of ICU acquired weakness.

\section{Materials and Methodology}

Ethical clearance was obtained from the institutional ethical committee. The study was carried out in the "CORONA CARE UNIT" which is the ICU of the Krishna Hospital and Medical Research Centre, Karad dedicated for the care and treatment of COVID-19 patients. 32 patients who were admitted in ICU were recruited for the study. Written as well as verbal informed consent was obtained from each subject. The pre-intervention data was recorded prior to the commencement of the study. Subjects with or without ongoing $\mathrm{O}_{2}$ therapy, having $\mathrm{SPO}_{2}>93 \%$ and having stable vitals were included in the study. Those with co-morbidities like HTN, Diabetes as well as those who were disoriented or uncooperative were excluded. The Twist and Raise walking technique was demonstrated to each patient.

\section{Procedure}

\subsection{The Twist and Raise Walking Technique}

The twist and raise walking technique consists of normal ambulation combined with trunk rotation as well as upper limb overhead movements. The technique consists of normal step by step walking combined with dynamic movements of trunk as well as upper limbs.
The starting position is standing upright. The individual is asked to take a step forward. Then he/she is asked to twist the trunk first to left side with both shoulder abducted to $90^{\circ}$.

Once the complete available range of lateral rotation (twisting) of the trunk is achieved by the individual, he/ she is instructed to further abduct both the hands till the palms approximate each other.

Once both the palms have completely touched each other then he/she has to lower both the upper limbs to $90^{\circ}$ of abduction, rotate back to starting position of the trunk, take both the upper limbs back to anatomical position and step back the previously advanced lower limb.

Same procedure has to be repeated with the other lower limb.

For beginners, the technique can be performed as 3 sets of 10 repetitions which can be increased in terms of no. of repetitions and hold-period depending upon the tolerance of the patient.

Each subject was asked to exercise using the prescribed technique for 5 minutes three times a day. The primary investigator was posted at the corona care unit as "Intensive Unit Physiotherapist" for a period of 7 days. Baseline data for demographic characteristics like age, gender and BMI was recorded. Post-intervention data for outcome measures like Incentive Spirometry (hold period at various resistance levels) and Peak flow meter was recorded on the $8^{\text {th }}$ day of the study. Total duration of the study was 9 days.

\section{Statistical Analysis}

Statistical analysis of the raw data was done using the SPSS 21.0 software. The mean and standard deviation for various demographic characteristics like age and weight were obtained. The pre-test to post-test score comparison of various outcome measures like inspiratory hold duration and forced expiratory volume was done using paired-t test. Results were obtained.

\section{Results}

\subsection{Demographic Characteristics}

Table 1 depicts the demographic characteristics of the subjects enrolled for the study. As it was a single group pre-post experimental study, the subjects were assessed on a case to case basis. Emphasis was given to ensure 
Table 1. Demographic characteristics

\begin{tabular}{|c|c|c|c|}
\hline \multicolumn{2}{|c|}{$\begin{array}{c}\text { Gender Distribution } \\
(\mathbf{n}=32)\end{array}$} & \multirow{2}{*}{ Age } & Weight \\
\cline { 1 - 2 } Male & Female & & \\
\hline $18(56.3 \%)$ & $14(43.7 \%)$ & $46.12 \pm 16.38$ & $67.87 \pm 9.67$ \\
\hline
\end{tabular}

Table 2. Outcome measures pre-post comparison

\begin{tabular}{|l|c|c|}
\hline $\begin{array}{l}\text { Outcome } \\
\text { measures }\end{array}$ & $\begin{array}{c}\text { Inspiratory Hold } \\
\text { Duration }(\text { in sec) }\end{array}$ & $\begin{array}{c}\text { Forced Expiratory Volume } \\
(\text { lit } / \mathrm{sec})\end{array}$ \\
\hline Pre-test & $1.5 \pm 0.50$ & $334.68 \pm 147.43\left(\mathrm{p}<0.0001^{\star}\right)$ \\
\hline Post-test & $2.31 \pm 0.64$ & $371.56 \pm 146.42\left(\mathrm{p}<0.0001^{\star}\right)$ \\
\hline
\end{tabular}

equal gender distribution to increase the validity of the study. Out of total 32 subjects included in the study (N $=32$ ), 18 subjects were male whereas 14 subjects were female. The mean age of the subjects included in the study was $46.12 \pm 16.38$.

Table 2 depicts the pre-test as well as post test scores for both the outcome measures. Extremely statistical significant post-test scores were seen for both the outcome measures $\left(\mathrm{p}<0.0001^{\star}\right)$.

\section{Discussion}

The aim of the present study was to initiate or encourage early ambulation in prolong bed-ridden COVID-19 patients. The idea was to reduce the complications of ICU-acquired weakness.

A systematic review was carried out by Kalirathinam et al., $(2020)^{8}$ in an effort to provide a comprehensive physiotherapy protocol depending upon the stage of progression of the COVID-19 disease. It was highlighted that the physiotherapist should ideally initiate taking rehabilitation measures as early as possible. The minimum criteria being clinically stable patient, it was stated that early physiotherapy interventions like breathing techniques like pursed-lip or glossopharyngeal breathing to reduce dyspnoea, tracheo-bronchial clearance and skeletal muscle training should be contraindicated during the acute phase of the disease. But as the list of symptoms can't be generalized and so does the physiotherapy rehabilitative approach, it is only experimentation which would provide more precise rehabilitative approach from physiotherapy point of view.

In this study, transition from sitting in the bed to standing and walking was made possible by the twist and raise walking technique. The unique factor which makes the technique more potent and beneficial especially in improving the respiratory parameters is the component of trunk rotation along with upper limb movements. The trunk rotation helped to stretch the accessory muscles of respiration like internal obliques, external obliques, intercostals muscles etc which might have added in extra space for the expansion of the rib cage thus facilitating improved lung expansion and in turn improved inspiratory hold capacity as well as forced expiratory capacity.

The possible mechanism behind the extremely significant improvement seen in Forced Expiratory Volume might be the trunk rotation component which might have resulted in increasing the rib-cage movement and thus providing more space to accommodate expansion of the lungs.

\section{Conclusion}

The innovative Twist and Raise Walking Technique can be administered safely as a rehabilitative exercise therapy to COVID-19 positive patients in order to improve the respiratory capacity and thus in order to minimize the complications of ICU-acquired weakness. It also initiates early ambulation in a bed-ridden patient.

\section{References}

1. Del Rio C, Malani PN. Novel Coronavirus-important information for clinicians. JAMA. 2020; 323:1039-40. PMid: 32022836. https://doi.org/10.1001/jama.2020.1490

2. World Health Organization. Coronavirus disease 2019 (COVID-19) Situation Report 46, 2020.

3. Kiekens C, Boldrini P, Andreoli A, Avesani R, Gamna F, Grandi M, Lombardi F, Lusuardi M, Molteni F, Perboni A, Negrini S. Rehabilitation and respiratory management in the acute and early post-acute phase. "Instant paper from the field" on rehabilitation answers to the COVID-19 emergency. Eur J Phys Rehabil Med. 2020; 56(3):323-6. https://doi.org/10.23736/S1973-9087.20.06305-4

4. Kress JP, Hall JB. ICU-acquired weakness and recovery from critical illness. N Engl J Med. 2014; 370(17):1626-35. PMid: 24758618. https://doi.org/10.1056/NEJMra1209390

5. Zhonghua Jie He He Hu Xi Za Zhi. Chinese association of rehabilitation medicine; Respiratory rehabilitation committee of Chinese Association of Rehabilitation Medicine; Cardiopulmonary rehabilitation Group of Chinese Society of Physical Medicine and Rehabilitation. 
Recommendations for respiratory rehabilitation of COVID-19 in adult. (Chinese Journal of Tuberculosis and Respiratory Diseases), 2020 Mar 3; 43(0):E029. doi: 10.3760/cma.j.cn112147-20200228-00206.

6. Thomas P, Baldwin C, Bissett B, Boden I, Gosselink R, Granger CL, Hodgson C, Jones AY, Kho ME, Moses R, Ntoumenopoulos G, Parry SM, Patman S, van der Lee L. Physiotherapy management for COVID-19 in the acute hospital setting: Clinical practice recommendations. Journal of Physiotherapy. 2020; 66(2):73-82. PMid: 32312646 PMCid: PMC7165238. https://doi.org/10.1016/j. jphys.2020.03.011

7. Lazzeri M, Lanza A, Bellini R, Bellofiore A, Cecchetto S, Colombo A, D'Abrosca F, Del Monaco C, Gaudiello G,
Paneroni M, Privitera E, Retucci M, Rossi V, Santambrogio M, Sommariva M, Frigerio P. Respiratory physiotherapy in patients with COVID-19 infection in acute setting: a position paper of the Italian Association of Respiratory Physiotherapists (ARIR). Monaldi Arch Chest Dis. 2020 Mar 26; 90(1):163-8. https://doi.org/10.4081/ monaldi.2020.1285

8. Kalirathinam D, Guruchandran R, Subramani P. Comprehensive physiotherapy management in COVID-19 - A narrative review. Scientia Medica. 2020; 30(1):38030. https://doi.org/10.15448/1980-6108.2020.1.38030 